\title{
Confinement Loss and GVD for HF and AHAOF by FEM
}

\author{
Akbar Zende hnam*, Mahmoud Mirzaei, Razieh Solgi \\ Department of Physics, Faculty of Science, Arak University, Arak, 38156-8-8349, Iran
}

\begin{abstract}
An analysis of the confinement loss and Group Velocity Dispersion (GVD) due to their importance is performed by means of the fin ite element method for two kinds of fibers, an Air-Hole Assisted Optical Fiber (AHA OF) and a Holy Fiber (HF). Both structures are based on a subset of a triangular array of cy lindrical air holes; the cross sections of these inclusions are circular and the refractive index of the AHAOF core is higher than clad, and a missed central hole forms the HF core. The group velocity dispersion shows different behaviours in different ranges of wavelength also numerical results which we obtained agree well with the results of the other reported methods for HF dispersion but the results of the AHAOF dispersion were different fro $\mathrm{m}$ the outcome of the fin ite difference method for this fiber. The nu merical results indicate that confinement loss increases with increasing wavelength and they show that the confinement loss of AHAOF is higher than HF because the smaller air holes in structure of HF can confine fields in the core region more than AHAOF.
\end{abstract}

Keywords Finite Element Method, Group Velocity Dispersion, Photonic Crystal Fibers

\section{Introduction}

Photonic crystal fibers (PCFs) guide the electro magnetic field by means of an arrangement of air holes that run down the entire fiber length. In the holy fibers the air holes reduce the average refractive index around the solid core and guidance can be ascribed by total internal reflection. Conversely in the photonic band gap fibers the average core index could be lower than the average cladding index and the guidance is due to the photonic band gap in the transverse direction. One of the appealing characteristics of such fibers is that their dispersion and modal properties can be controlled significantly by varying the size (diameter) of the air-holes, their nu mber, and their positions [1]. In both cases a lossless propagation is possible only if the air hole arrangement is of infinite extent and, of course, if a lossless material is used. A fiber can be considered as confinement lossless if the field leakage is negligible with respect to the material losses. To investigate leaky modes properties, in order to find guidelines for the design of lossless fibers, numerical models with proper boundary conditions must be adopted. In fact, periodic boundary conditions or perfect electric or magnetic conductor boundary conditions which usually are employed in the numerical models affect the transverse Poynting vector flow of the numerical solutions.

* Corresponding author:

a-zendehnam@araku.ac.ir (Akbar Zendehnam)

Published online at http://journal.sapub.org/optics

Copyright (C) 2012 Scientific \& Academic Publishing. All Rights Reserved
Among the full vectorial methods used in modelling PCFs [2-7], the finite-element method (FEM) is particularly effective for handling curved interfaces with high accuracy, and it is obviously a good choice for the analys is of combined circular and elliptical shapes. The numerical method used in this study is FEM which is adequate and suitable for the analysis of general dielectric waveguide geometries. Although it has complicated equations to solve and needs to spend long time, it is more accurate than the other methods[8-11]. It has been already success fully applied to investigate dispersion properties of triangular and cobweb PCFs[12]. The fiber cross-section representation is very accurate as the domain is divided into subdomains with triangular or quadrilateral shapes, where any refractive index profiles can be properly represented. Applying the variational FEM procedure to the curl-curl equation for magnetic field $\mathrm{H}$, one obtains an eigenvalue equation (1) [12]:

$$
\left([P]-\beta^{2}[Q]\right)\{H\}=0
$$

where $\{H\}$ is the global magnetic field vector, $\beta$ is the propagation constant, and $[P]$ and $[Q]$ are both sparse matrices. The symmetry of the fiber cross-section is exploited to reduce CPU time and memory requirements. Solving the eigenvalue equation yields the effective indices of guided modes $n_{\text {eff }}=\beta / k_{0}$, where $k_{0}=2 \pi / \lambda$ is the wave vector in free space.

\section{Results and Discussion}

\subsection{Air-Hole-Ass is ted Optical Fiber (AHAOF)}




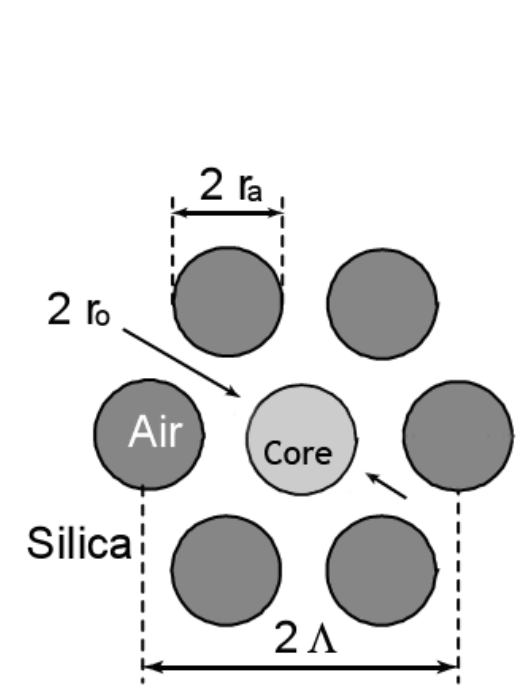

(a)

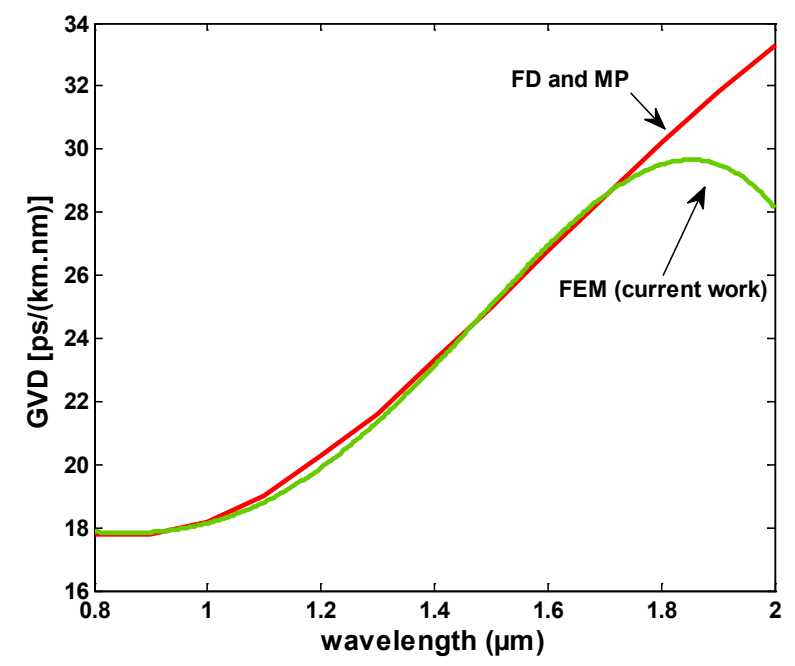

(c)

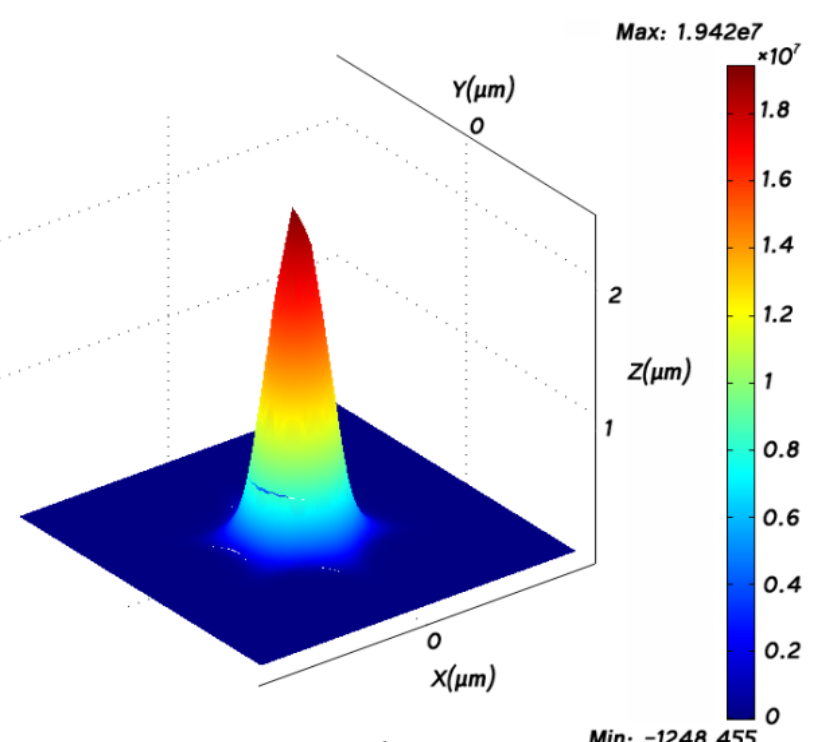

(b)

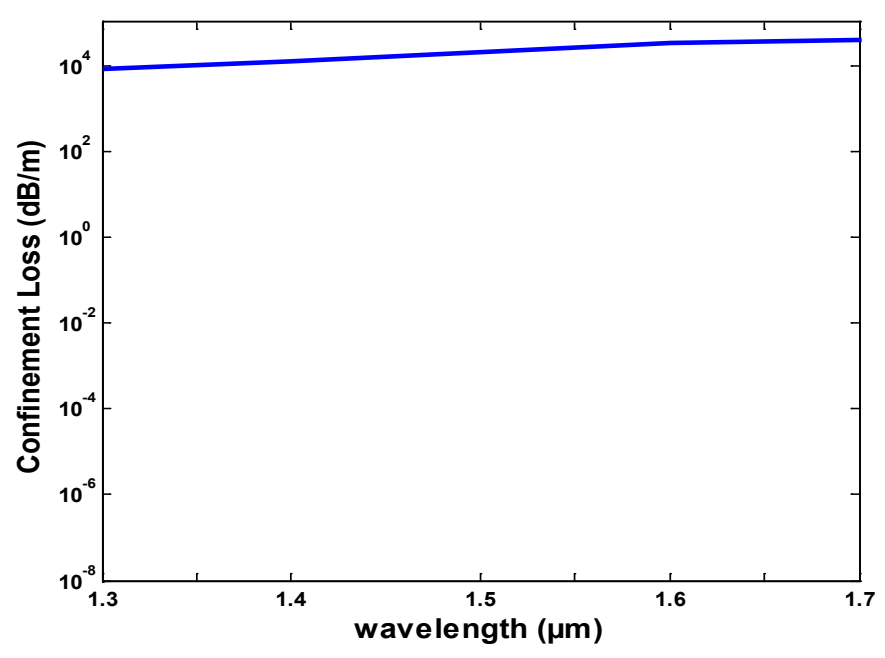

(d)

Figure 1. (a)Schematic of an air-hole assisted optical fiber(Core index 1.45, silica cladding index1.42, $r_{0}=2 \mu \mathrm{m}, r_{a}=2 \mu \mathrm{m}$, $\lambda=2 \mu \mathrm{m}$ ); (b)Magnetic field plot of the fundamental mode (y-polarization); (c)Calculated GVD curve (material dispersion not considered); (d)Calculated confinement loss curve, against wavelengths

An (AHAOF) [13] is a microstructured fiber that has air holes surrounding the raised-index guiding core (see Figure 1(a)). One interesting aspect of the AHAOF is that it has easily tailorable dispersion and polarization properties while having low transmission loss comparable to conventional optical fibers. The modal characteristics of AHAOF can be accurately obtained by using the multipole [MP] method[4]. Here the FEM is used to examine this fiber and compare the results with those by the multipole analysis and finite different [FD] methods. In Figure 1(b) the magnetic field plot of the y-polarized fundamental mode is given. In Figure 1(c) also is shown the group velocity dispersion (GVD) curves against wavelength calculated from the results of FEM and the FD and the MP methods. The agreement is excellent. In the calculation of the GVD graphs, material dispersion is not considered, which is dominant at shorter wavelengths but for higher wavelengths it is assumed to be constant, i.e., $n_{\text {core }}=1.45, n_{\text {clad }}=1.42$ and $\mathrm{n}=1$ (for air holes). While it is trivial to take into account the material dispersion by changing the refractive index at each wavelength, small differences in GVD between different approaches are better appreciated by excluding material dispersion. As for this figure, the GVD is constant at low wavelengths $(\lambda<1 \mu \mathrm{m})$ and afterward increases linearly with a sharp slope up to about $1.7 \mu \mathrm{m}$, then the plot related to the FEM starts to decrease with wavelength but for other methods continues to increase. It is predicted that this behaviour is because of the dimension of the air holes are getting close to wavelengths (for $\lambda>1.7 \mu \mathrm{m})$. In Figure 1(d) the variation of the confinement loss against wavelength $(1300-1700 \mathrm{~nm})$ for AHAOF is given, which is increasing linearly with smooth slope.

\subsection{Holy Fiber (HF)}


A HF (Figure 2(a), triangular array with circular air holes is shown) is analysed with the FEM. In holey optical fibers, a mode is guided if its effective mode index satisfies $n_{F S M}<n_{\text {eff }}<n_{S i}[14]$, where $n_{s i}$ is the refractive index of silica, and $n_{F S M}$ is the effective index of the fundamental space-filling mode (FSM)[14, 15] of the infinite periodic cladding. $n_{F S M}$ is an important parameter in the effective index model of ho ley fibers, which can give estimation of the number of guided modes and the effective mode areas[16, 17]. The field plot for the fundamental mode is shown in Figure 2(b). In Figure 2(c) the curves of the calculated GVD versus wavelength from FEM and FD mode solvers and the localized function method are given. It shows that calculated GVD for all of the mentioned methods is increases with an almost sharp slope up to about $1 \mu \mathrm{m}$ wavelength and after that is roughly constant up to about $1.4 \mu \mathrm{m}$ and then start to re- duce sharply. The plots reduction at higher wavelengths are not in a excellent agreement and FD method gives a lower value for GVD at $\lambda \cong 2 \mu \mathrm{m}$. The confinement loss versus wavelength for this fiber is given in Figure 2(d); which is almost linear and increases with wavelength. The confinement loss of HF is less than AHAOF also its plot slope is lower in the same ranges of wavelength $(1300-1700 \mathrm{~nm})$, so it is more beneficial to use them (HF) in some applications which need constant or low confinement loss. Increasing the number of air hole rings affects the confinement loss of microstructure fibers generally. Although the amount of confinement loss is reduced by increasing the number of a ir hole rings(is illustrated in[18]) plot gradient of curves increases thus using the holy fibers with less number of ring holes even with higher confinement loss is more beneficial.
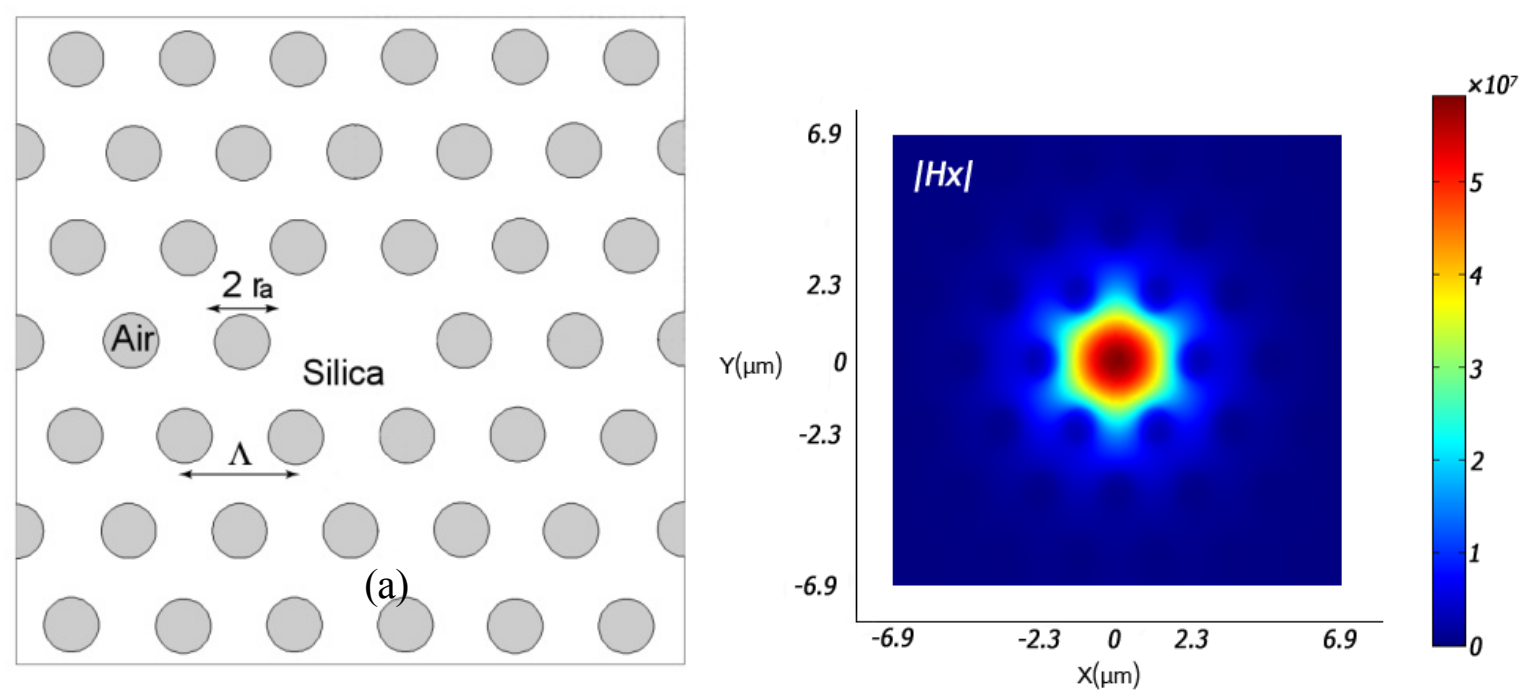

(b)

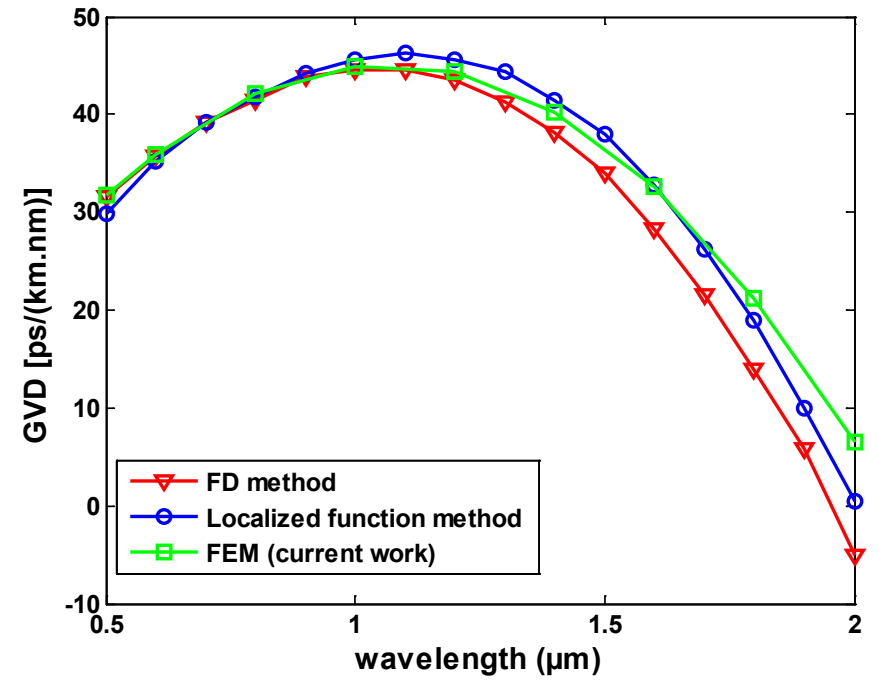

(c)

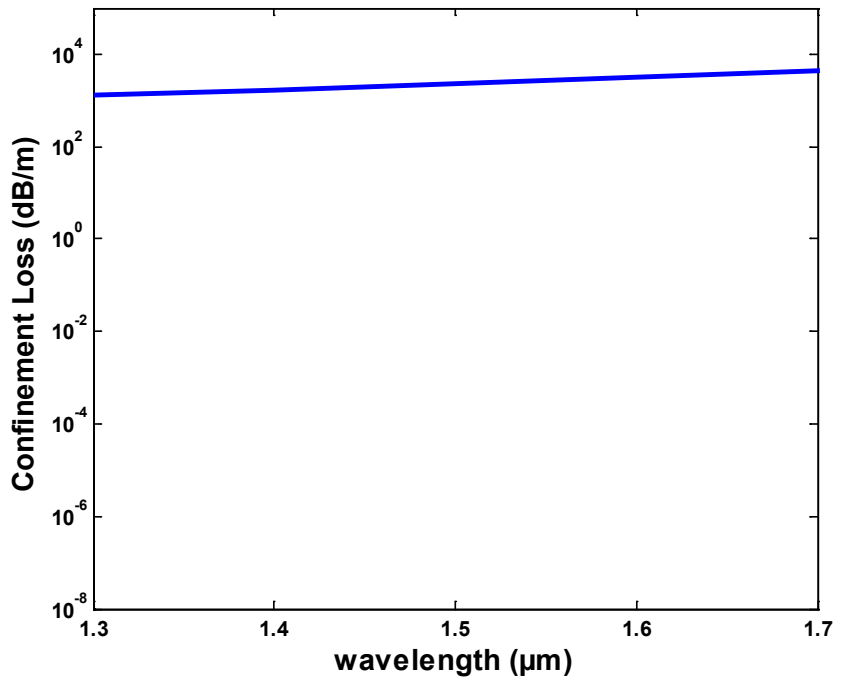

(d)

Figure 2. (a)Calculation window of the holey fiber $(\vDash 2.3 \mu \mathrm{m}, r=5 \mu \mathrm{m})$, silica refractive index of 1.45 is assumed at all wavelengths); (b)Field plots of the y-polarized fundamental mode; (c)Calculated GVD curves; (d)Calculated confinement loss curve, versus wavelength 


\section{Conclusions}

The confinement loss and group velocity dispersion of two types of microstructured optical fibers against wavelength were demonstrated by finite element method successfully. The confinement loss of type1 (AHAOF) is higher than type 2 (HF) because the smaller a ir holes in structure of type 2 can confine fields in the core region more than type1. The calculated GVD curves for both structures are in complete adjustment to the other calculated methods. The confinement loss is a linear function of wavelength in both structures (in the communication wavelength range $1300 \mathrm{~nm}<\lambda<1700 \mathrm{~nm}$ ).

\section{REFERENCES}

[1] S.M. Abdur Razzak, M. A. Rashid, Y Namihira, and A. Sayeem, "Group Velocity Dispersion Management of Microstructure Optical Fibers," International Journal of Electrical and Information Engineering, vol. 4, no. 3, 2010.

[2] A. Ferrando, E. Silvestre, J. J. Miret, P. Andrés, and M. V. Andrés, "Full-vector analysis of a realistic photonic crystal fiber," Opt. Lett., vol. 24, no. 5, 1999.

[3] D. Mogilevtsev, T. A. Birks, and P. St. J. Russell, "Localized function method for modeling defect modes in 2-D photonic crystals," J. Lightwave Technol., vol. 17, no. 11, 1999.

[4] Z. Zhu and T. G. Brown, "Multipole analy sis of hole-assisted optical fibers," Opt. Commun., vol. 206, no. 4-6, 2002.

[5] Z. Zhu and T. G. Brown, "Full-vectorial finite-difference analy sis of microstructured optical fibers," Opt. Express, vol. 10 , no. $17,2002$.

[6] F. Brechet, J. Marcou, D. Pagnoux, and P. Roy, "Complete analysis of the characteristics of propagation into photonic crystal fibers, by the finite element method," Opt. Fiber Technol., vol. 6, no. 2, 2000.

[7] Z. Zhu and T. G. Brown, "Stress-induced birefringence in microstructured optical fibers," Opt. Lett., vol. 28, no. 23, 2003.

[8] A. Cucinotta, S. Selleri, L. Vincetti, and M. Zoboli, "Holey fiber analysis through the finite-element method,'IEEE Photonic Tech. L., vol. 14, no. 11, 2002.

[9] D. Mao, C. Guan, and L. Yuan, " $1 \times 4$ coupler based on all solid five-core photonic crystal fibers," Opt. Commun., vol. 284, no. 19, 2011.

[10] A. Ademgil, and S. Haxha, "Bending insensitive large mode area photonic crystal fiber," Optik, vol. 122, no. 21, 2011.

[11] S. E. Kim, B. H. Kim, C. G. Lee, S. Lee, K. Oh, and C. S. Kee, "Elliptical defected core photonic crystal fiber with high birefringence and negative flattened dispersion," Opt. Express, vol. 20 , no. 2 , p. 1385-1393, 2012.

[12] T. Hasegawa, E. Sasaoka, M .Onishi, M. Nishimura, Y. Tsuji and M. Koshiba, "Hole-assisted light guide fiber for large anomalous dispersion and low optical loss," Opt. Express, vol. 9, no. 13, 2001.

[13] M.Midrio, M. P. Singh, and C. G. Someda, "The space filling mode of holey fibers: an analytical vectorial solution," J. Lightwave Technol., vol. 18, no. 7, 2000.

[14] Z. Zhu and T. G. Brown, "Analy sis of the space filling modes of photonic crystal fibers," Opt. Express, vol. 8, no. 10, 2001.

[15] N. A. Mortensen, "Effective area of photonic crystal fibers," Opt. Express, vol. 10, no. 7, 2002.

[16] J. C. Knight, T. A. Birks, P. St. J. Russell and J. P. deSandro, "Properties of photonic crystal fiber and the effective index model," J. Opt. Soc. Am. A, vol. 15, no. 3, 1998.

[17] D. Ferrarini. Luca Vincetti. M. Zoboli. A. Cucinotta. and S. Selleri, "Leakage properties of photonic crystal fibers," Opt. Express, vol. 10, no. 23, 2002.

[18] Kenta Kaneshima, Yoshinori Namihira, Nianyu Zou, Hiroki Higa, Yasunori Nagata, "Numerical Investigation of Octagonal Photonic Crystal Fibers with Strong Confinement Field," IEICE Trans. Electron. E89-C, vol. 6, p. 830-837, 2006. 